This item was submitted to Loughborough's Research Repository by the author.

Items in Figshare are protected by copyright, with all rights reserved, unless otherwise indicated.

\title{
Exact eigensolution of a class of multi-level elastically connected members
}

PLEASE CITE THE PUBLISHED VERSION

http://dx.doi.org/10.1016/j.engstruct.2017.03.059

\section{PUBLISHER}

(c) Elsevier

VERSION

AM (Accepted Manuscript)

\section{PUBLISHER STATEMENT}

This work is made available according to the conditions of the Creative Commons Attribution-NonCommercialNoDerivatives 4.0 International (CC BY-NC-ND 4.0) licence. Full details of this licence are available at: https://creativecommons.org/licenses/by-nc-nd/4.0/

\section{LICENCE}

CC BY-NC-ND 4.0

\section{REPOSITORY RECORD}

Howson, W.P., and Andrew Watson. 2017. "Exact Eigensolution of a Class of Multi-level Elastically Connected Members". figshare. https://hdl.handle.net/2134/24881. 


\title{
Exact Eigensolution of a class of multi-level elastically connected members
}

\author{
W.P. Howson ${ }^{1}$ and A. Watson $*^{2}$ \\ ${ }^{1}$ Independent Consultant, Gwanwyn, Craig Penlline, CF71 7RT, UK \\ ${ }^{2}$ Department of Aeronautical and Automotive Engineering, Loughborough University, UK
}

*Contact information for corresponding author A Watson

Email: $\quad$ a.watson@lboro.ac.uk

Post address: Department of Aeronautical and Automotive Engineering

Stewart Miller Building

Loughborough University

Leicestershire LE11 3TU

Final version as changed to take account of the referee's comments $27^{\text {th }}$ January 2017 


\section{ABSTRACT}

Attention is given to determining the exact natural frequencies and modes of vibration of a class of structures comprising any number of related parallel members that are connected to each other, and possibly also to foundations, by uniformly distributed elastic interfaces of unequal stiffness. The members themselves are considered to have a uniform distribution of mass and stiffness and account can be taken of additional point masses and spring supports. The formulation is general and applies to any structure in which the motion of the component members is governed by a second order Sturm-Liouville equation. Closed form solution of the governing differential equations leads either, to a series of exact substitute systems that are easy to solve through a stiffness approach and which together yield the complete spectrum of natural frequencies and corresponding mode shapes of the original structure, or to simple exact relationships between the natural frequencies corresponding to coupled and uncoupled motion that enable hand solution of the more standard problems to be achieved. An appropriate form of the Wittrick-Williams algorithm is presented for converging on the required natural frequencies to any desired accuracy with the certain knowledge that none have been missed. Examples are given to confirm the accuracy of the approach and to indicate its range of application.

Keywords: Exact eigensolution, elastically connected members, dynamic stiffness, WittrickWilliams algorithm.

\section{Introduction}

The dynamics of a family of simple, but extremely useful structural elements is governed by a second order Sturm-Liouville equation. This equation allows for the uniform distribution of mass and stiffness and enables the motion of strings and shear beams, together with the axial and torsional motion of bars to be described exactly. As a result, each member type in this family has been treated exhaustively when considered as a single member or when joined contiguously to others, e.g. Rao [1]. However, when such members are linked in parallel by uniformly distributed elastic interfaces, their complexity becomes significantly more intractable and it is this class of structures that has led to renewed interest and which forms the basis of the work that follows.

Research in the general area of spring linked members has primarily focussed on flexural beam systems and a substantial volume of work is available in the literature. Since this does not impinge meaningfully on the work that follows, the literature will not be reviewed, except to note an early paper that deals with comparable issues to those developed herein [2], together with a recent paper that cites a good selection of recent work [3]. In contrast, relatively little work has been undertaken on the class of structures considered below. Perhaps most interest has been directed towards double string systems, which have generated a number of papers [4-7], as have problems associated with the longitudinal motion of spring linked bars [8-11]. In contrast, the torsional vibration problem has seen less activity [12-13] and there is little evidence of any related work on the motion of beams vibrating in shear. The latter point is particularly surprising for the case when beams vibrate in pure shear, since it is a common occurrence in beams of small aspect ratio or when vibrating at high frequencies [14].

The theory developed in Section 2 of this paper establishes a unified approach to the exact solution of a class of structures comprising related parallel members of the type described above, that are linked to each other and possibly foundations in a complex way by uniformly 
distributed elastic interfaces of unequal stiffness. Any number of members can be accommodated, together with any possible combination of allowable boundary conditions.

Initially, differential equations governing the coupled motion of the system are developed from first principles. These are organised into the form of a generalised linear symmetric eigenvalue problem, from which a family of uncoupled differential operators can be established. These operators define a series of exact substitute systems that together describe the complete motion of the original structure. These equations can then be used in either of two ways. In their most powerful form they can be developed into exact dynamic stiffness matrices that enable all the powerful features of the finite element method to be utilised. This subsequently enables sets of members carrying point masses and subject to point spring supports to be analysed easily. Alternatively, the equations are able to yield an exact relational model that links any uncoupled frequency of an original member to the corresponding set of coupled system frequencies. This approach enables 'back of the envelope calculations' to be undertaken quickly and efficiently. It is worth noting in connection with this, that the member theory has been developed in the context of linked shear beams. This leads directly to a practical application of the method in which an approximate analysis can be undertaken to assess the vibrational characteristics of sway frames either linked together or additionally linked to shear cores. This can be achieved using well established simplification procedures that reduce multi-bay, multi-storey sway frames to equivalent one bay frames and then to simpler global models that can often retain sufficient accuracy for preliminary analysis and design procedures [15-17]. Additional practical applications, including those for the member types listed in Table B1, include, where appropriate, the analysis of: single members on a distributed elastic foundation; laminated members formed from alternate layers of adhesive and laminate; and structural systems formed from members linked by thin metal plate. Furthermore, it can be demonstrated [18] that using distributed elastic interfaces can yield approximate solutions to good accuracy when the actual connection comprises a sufficient number of regularly spaced, point connections, thus enabling: single members on elastic pad foundations; complex cable or beam trusses; and plane grillages, to be analysed simply and efficiently.

The paper is concluded with a number of examples that confirm the accuracy of the proposed method and indicate its range of application.

\section{Theory}

\subsection{A single set of members}

The equation of motion for a typical member, $i$, in Fig. 1 below and its corresponding constitutive equation are easily shown to be

$$
\partial q_{i} / \partial x+k_{i} v_{i-1}-\left(k_{i}+k_{i+1}\right) v_{i}+k_{i+1} v_{i+1}=m_{i} \partial^{2} v_{i} / \partial t^{2}
$$

and

$$
q_{i}=A_{s} G_{i} \partial v_{i} / \partial x
$$

where $q$ is the shear force, $m$ is the mass / unit length, $k$ is the stiffness / unit length of an elastic interface, $A_{s} G$ is the effective shear rigidity and the subscripts refer to the respective components shown in Fig. 1. Substituting Eq.(2) into Eq.(1), imposing the assumption of 
harmonic motion and introducing the non-dimensional parameter, $\xi=x / L$, enables Eqs.(1) and (2) to be written as

$$
k_{i} V_{i-1}-\left(k_{i}+k_{i+1}\right) V_{i}+k_{i+1} V_{i+1}+r_{i}\left(D^{2}+\gamma_{i} \omega^{2}\right) V_{i}=0
$$

and

$$
Q_{i}=r_{i} L d V_{i} / d \xi
$$

where

$$
D^{2}=d^{2} / d \xi^{2}, \quad r_{i}=A_{s} G_{i} / L^{2}, \quad \gamma_{i}=m_{i} / r_{i}
$$

$L$ is the length of all members comprising the set, $\omega$ is the circular frequency and the remaining upper case characters are amplitudes of their lower case counterparts. It is now assumed that $m_{i} / A_{s} G_{i}$ is constant for all $i$ and hence that

$$
\gamma_{i}=\gamma=m L^{2} / A_{s} G=\text { constant }
$$

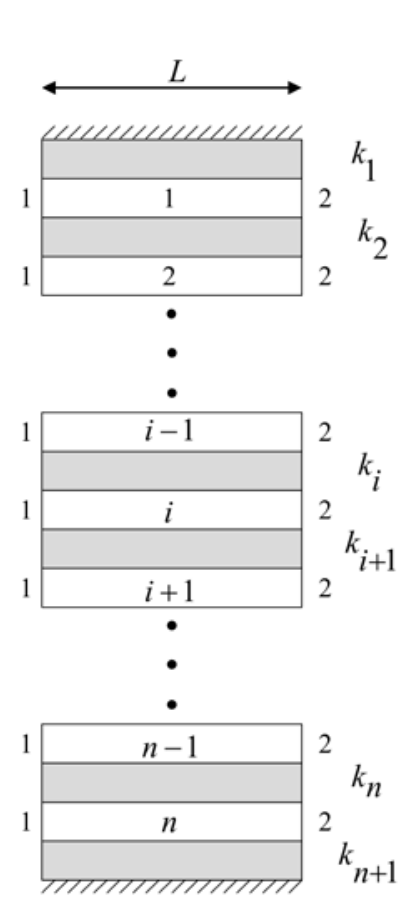

(a)

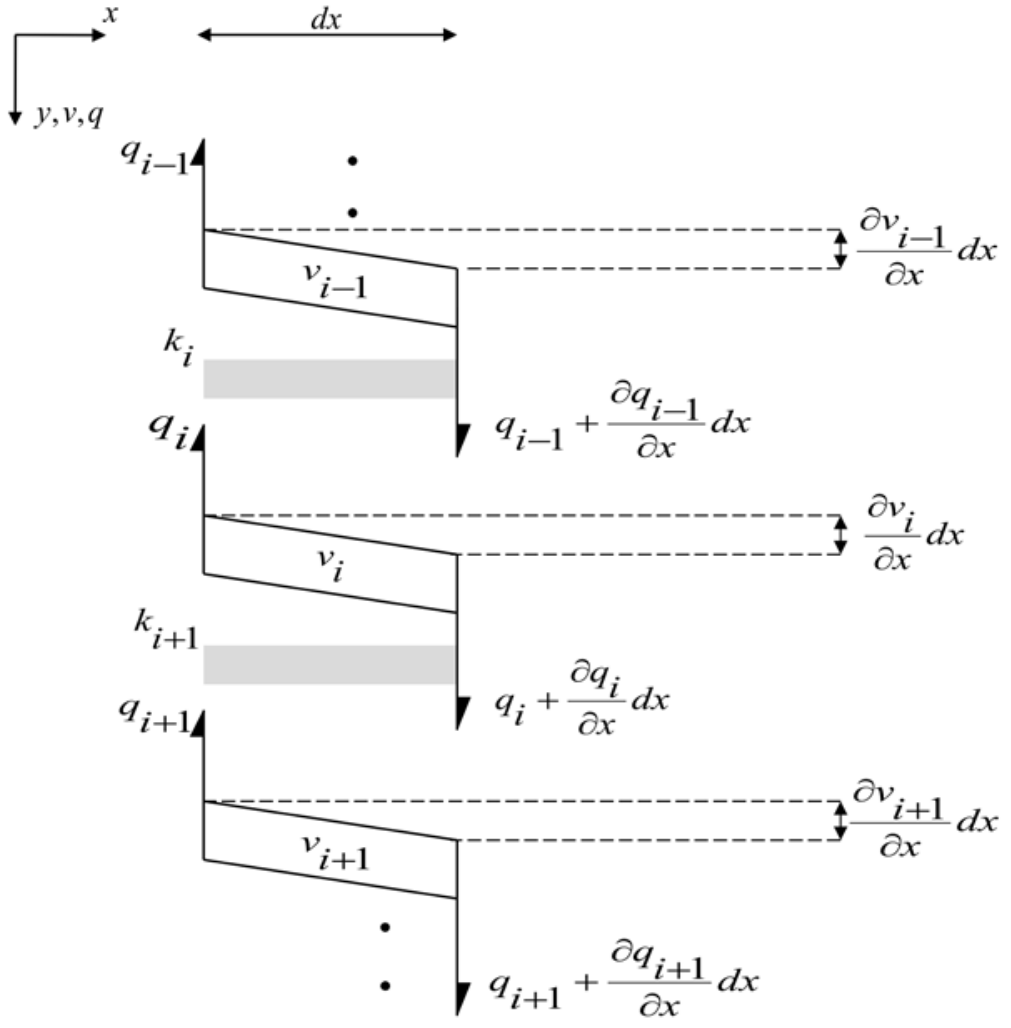

(b)

Fig. 1. Notation and positive sign convention for elastically connected members: (a) The complete set; (b) An elemental length of a typical member and its neighbours. 
This enables the governing equations for the first $(i=1)$, last $(i=n)$ and typical members to be written as

$$
\begin{gathered}
\left(k_{1}+k_{2}\right) V_{1}-k_{2} V_{2}-r_{1} \lambda V_{1}=0 \\
-k_{n} V_{n-1}+\left(k_{n}+k_{n+1}\right) V_{n}-r_{n} \lambda V_{n}=0 \\
-k_{i} V_{i-1}+\left(k_{i}+k_{i+1}\right) V_{i}-k_{i+1} V_{i+1}-r_{i} \lambda V_{i}=0
\end{gathered}
$$

respectively, where

$$
\lambda=D^{2}+\gamma \omega^{2}
$$

and $k_{1}$ and $k_{n+1}$ can be zero or non-zero in any combination, thus defining the longitudinal boundary conditions in Fig. 1(a). In addition, it is instructive to consider one further longitudinal boundary condition in which the boundary interfaces, $k_{1}$ and $k_{n+1}$, in Fig. 1(a) are removed and replaced by an elastic interface that connects the first and last members [2]. In order to achieve this, let the effective stiffness/unit length of the new connective interface be $k_{c}$. Eqs.(7a) and (7b) can then be replaced by

and

$$
\left(k_{c}+k_{2}\right) V_{1}-k_{2} V_{2}-k_{c} V_{n}-r_{1} \lambda V_{1}=0
$$

respectively.

$$
-k_{c} V_{1}-k_{n} V_{n-1}+\left(k_{n}+k_{c}\right) V_{n}-r_{n} \lambda V_{n}=0
$$

Eqs.(7) and (9) enable a complete set of equations to be assembled for an $n$ level system, as indicated by Eq.(10a)

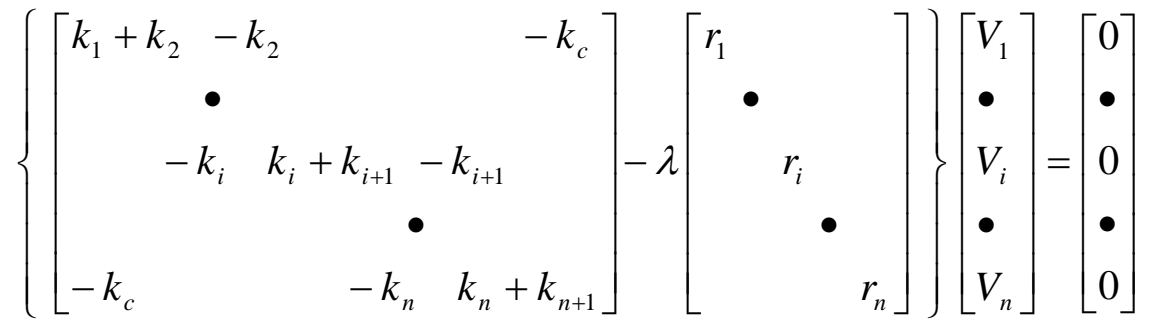

where zeros have been omitted for clarity, $k_{1}=k_{n+1}=k_{c}$ for the circulant case and $k_{c}=0$ otherwise. Moreover it can be seen that the circulant option is a special case of a more general relationship in which any member could additionally be connected to any other member or directly to a foundation. Such cross-connections can be carried out to any level of complexity and only has the effect of filling out the elastic interface matrix, while leaving the underlying theory unaltered. Eq.(10a) can then be written for any appropriate formulation as

$$
(\mathbf{k}-\lambda \mathbf{r}) \mathbf{V}=\mathbf{0}
$$

The form of Eqs.(10) is that of a generalized symmetric linear eigenvalue problem, for which a number of standard routines are available for calculating the eigenvalues, $\lambda$, and corresponding modal vectors, $\mathbf{V}$. 


\subsection{Substitute systems}

The $n$ values of $\lambda$ that satisfy Eqs.(10) define a family of second order differential operators that satisfy the original problem and which are given by Eq.(8) as

$$
D^{2}+\gamma \omega^{2}=\lambda_{i} \quad i=1,2, \ldots \ldots n
$$

Eq.(11) can be assigned a physical context by noting that it is a property of such differential operators that they can be written as

and hence that

$$
\left(D^{2}+\gamma \omega^{2}\right) V_{i}=\lambda_{i} V_{i} \quad i=1,2, \ldots n
$$

$$
\left(D^{2}+\chi_{i}^{2}\right) V_{i}=0 \quad i=1,2, \ldots n
$$

where

$$
\chi_{i}^{2}=\gamma \omega^{2}-\lambda_{i}
$$

and $V_{i}$ is a typical lateral displacement amplitude. In this case, each equation now describes the free vibration of a single unified member, but supported on a Winkler foundation of different magnitude in each case. Eqs.(13) therefore represent $n$ substitute systems, each of which yield an infinite number of frequencies that, when arranged in ascending order, comprise the complete spectrum of frequencies of the original problem. It therefore follows that the fundamental frequency of the original problem is given by the single substitute system that yields the lowest frequency, namely the one that incorporates the lowest linear eigenvalue derived from Eqs.(10).

An exact stiffness approach (exact finite element) is now adopted to solve the $i^{\text {th }}$ substitute system. Solutions of the form $V_{i}=A e^{s \xi}$ are first assumed, from which Eq.(13) yields the characteristic equation and the corresponding general solution as, respectively,

$$
s^{2}+\chi_{i}^{2}=0 \quad \text { and } \quad V_{i}=A_{1} C_{i}+A_{2} S_{i}
$$

where

$$
C_{i}=\cos \chi_{i} \xi \text { and } S_{i}=\sin \chi_{i} \xi \text { for } \chi_{i}^{2}>0
$$

and

$$
C_{i}=\cosh \chi_{i} \xi \text { and } S_{i}=\sinh \chi_{i} \xi \text { for } \chi_{i}^{2}<0
$$

Eqs.(4) and (16) in combination then yield the nodal values of $Q_{i}$ and $V_{i}$ when $\xi=0$ and 1 . Thus, changing from the local sign convention of Fig. 1(b) to the usual finite element convention in which the shear force on the left hand face is positive downwards, gives;

for $\xi=0, \quad Q_{i 0}=-Q_{i}$ and $V_{i 0}=V_{i} ; \quad$ and for $\xi=1, \quad Q_{i 1}=Q_{i}$ and $V_{i 1}=V_{i}$

Imposing these boundary conditions yields, after some manipulation, the required stiffness matrix for the $i^{\text {th }}$ substitute system as

$$
\left[\begin{array}{l}
Q_{i 0} \\
Q_{i 1}
\end{array}\right]=r_{i} L \frac{\chi_{i}}{\hat{S}_{i}}\left[\begin{array}{cc}
\hat{C}_{i} & -1 \\
-1 & \hat{C}_{i}
\end{array}\right]\left[\begin{array}{c}
V_{i 0} \\
V_{i 1}
\end{array}\right]
$$


where $\hat{C}_{i}$ and $\hat{S}_{i}$ are $C_{i}$ and $S_{i}$, respectively, when $\xi=1$. Finally Eq.(19a) can be written as

$$
\mathbf{Q}_{i}=\mathbf{K}_{s i} \mathbf{V}_{i}
$$

where $\mathbf{K}_{s i}$ is the $i^{\text {th }}$ substitute system matrix.

Identical boundary conditions are now imposed on each substitute system in turn by adding spring supports and/or nodal masses at both $\xi=0$ and $\xi=1$. There is no requirement for the masses to be the same at each end and the stiffnesses can be assigned any value between zero (free support) and $+\infty$ (clamped support). The required natural frequencies stemming from each of the $n$ substitute systems can then be converged upon to any desired accuracy using the Wittrick-Williams algorithm, described in Appendix A, which ensures that no natural frequencies can be missed. All the frequencies thus calculated are natural frequencies of the original system and can be arranged in ascending order to cover any frequency range of interest, which will be guaranteed to be fully populated if the highest frequency is bounded above in each of the substitute systems. A general procedure for establishing the corresponding mode shapes is deferred to Section 2.4.

\subsection{Linked sets of members}

The single set of members considered so far is now discretised into any number of sub-sets by cutting all members and elastic interfaces at identical points along their length. The number of members in each sub-set therefore remains equal to the number in the original set, but with each member having a constant, shorter length that can be unequal between sub-sets. Thus it would be possible to reconstitute the original structure by linking sub-sets together side by side. However, each sub-set can now be treated in an identical way to the original set and hence can be modelled as a series of substitute systems that can be described through a stiffness formulation. Thus linking sub-sets is equivalent to linking corresponding substitute systems from each sub-set, i.e. linking the $i^{\text {th }}$ substitute system from each set generates the $i^{\text {th }}$ extended substitute system, which can be achieved using standard techniques that are well established in the stiffness method of analysis. This results in $n$ extended systems, each of which can be solved independently and each of which now contain corresponding internal nodes. This clearly offers a simple procedure for recovering mode shapes, but more importantly enables point mass and stiffness to be applied at the nodes. Together these features give the ability to model structures that are genuinely complex along the length of the members, while remaining laterally repetitive.

\subsection{Mode shape recovery}

Consider one of the extended systems described above, from which a typical natural frequency has been determined. The corresponding system mode shape can then be generated by any appropriate method, such as that described by Howson [19]. The mode shape of each of the original members comprising the substitute system then share the mode shape of the substitute system, but with different amplitude. The unique member mode shapes can therefore be found by scaling the substitute system mode shape by the corresponding element of the eigenvector stemming from the appropriate linear eigenvalue problem. 


\subsection{Frequency relationships}

The theory defined above and its computer implementation in a stiffness formulation is at the heart of the present method. However, a useful frequency relationship that is exact and universally applicable is straightforward to develop.

\subsubsection{General}

Consider Eq.(11), which defines the governing differential operators for each of the $n$ substitute systems comprising the original problem and let the $j^{\text {th }}$ natural frequency stemming from the $i^{\text {th }}$ substitute system be

$$
\omega_{i, j} \quad i=1, \ldots n ; \quad j=1,2, \ldots \infty
$$

Eq.(11) then makes it is possible to write

$$
D^{2}+\gamma \omega_{l+k, j}^{2}-\lambda_{l+k}=D^{2}+\gamma \omega_{l, j}^{2}-\lambda_{l}
$$

and hence that

where

$$
\omega_{l+k, j}^{2}=\omega_{l, j}^{2}+\left(\lambda_{l+k}-\lambda_{l}\right) / \gamma
$$

$$
1 \leq l \leq n-1, \quad j=1,2, \ldots \infty \quad \text { and } \quad 2 \leq l+k \leq n
$$

Eq.(22) therefore relates any natural circular frequency in one substitute system to its corresponding frequency in any other substitute system.

\subsubsection{Hand calculation}

Eq.(22) has particular relevance when applied to hand calculation. In this role, practical considerations of time and effort will probably impose limitations on the problems attempted, with the result that its most likely application will be in the analysis of practical, repetitive structures in which all the members are identical with classical support conditions, there are no in-span point masses or point supports and all elastic interfaces are the same with no crossconnections. In such cases, hand solution using the theory below, aided by Table 1 and simple referenced formulae, enable exact solutions to be achieved extremely easily, as described in Section 2.6.2.

Consider Eq.(11) once more when

$$
\lambda_{i}=\lambda_{0}=0
$$

It can be seen that the equation then defines an additional 'substitute system' that yields the uncoupled natural frequencies of a component member. Eq.(22) therefore remains valid when Eq.(23) is modified as follows

$$
0 \leq l \leq n-1, \quad j=1,2, \ldots \infty \quad \text { and } \quad 1 \leq l+k \leq n
$$

In passing, it is interesting to note that the fundamental frequency of the set, $\omega_{1,1}$, only requires a knowledge of $\gamma, \omega_{0,1}, \lambda_{1}$ and the fact that $\lambda_{0}=0$. 
Table 1: Uncoupled natural frequencies of the set. The boundary conditions are denoted by C $=$ Clamped and $\mathrm{F}=$ Free. Due allowance should be made for rigid body modes and $k=j$ except for the pure shear case in which $k=0,1,2, \ldots \infty$.

\begin{tabular}{ccc}
\hline Boundary conditions & Condition in Eq.(19a) & $\begin{array}{c}\text { Frequency } \omega_{0, j} j=1,2, \ldots \infty \\
(\mathrm{rad} / \mathrm{sec})\end{array}$ \\
\hline C-C & $\sin \chi_{0}=0$ & $j \pi / \sqrt{\gamma}$ \\
C-F & $\cos \chi_{0}=0$ & $(2 j-1) \pi / 2 \sqrt{\gamma}$ \\
F-F & $\cos ^{2} \chi_{0}-1=0$ & $k \pi / \sqrt{\gamma}$ \\
\hline
\end{tabular}

\subsection{Analytical process}

\subsubsection{General}

The process of analysing a complex structure of the type described in Sections 2.1-2.3 can be carried out by following the steps described below. The process is clarified in Example 3.

- Discretise the structure so that the displacement vector will yield a sufficiently well defined mode shape.

-Develop $m$ appropriate sub-sets to satisfy the idealisation.

- For each sub-set:

- Solve the linear eigenvalue problem and store both the eigenvalue and the eigenvector (modal participation vector).

-Develop the $n$ substitute systems.

- Add corresponding substitute systems to form the $n$ extended substitute systems.

- For each extended system:

- Impose the boundary conditions and any other nodal mass or stiffness.

- Solve for the required number of natural frequencies and retrieve the corresponding extended system mode shapes.

- Retrieve the mode shapes of the original members by multiplying the extended system mode shapes by the appropriate components of the corresponding modal participation vector.

- Re-assemble the natural frequencies in ascending order and ensure that those of interest are bounded above in their respective substitute system.

\subsubsection{Hand calculation}

The process below generates all the natural frequencies and corresponding modes of vibration of the single member set proposed in Section 2.5.2, from a knowledge of the uncoupled frequencies of a component member.

-Determine the linear eigenvalues of the set, together with the corresponding eigenvectors (modal participation vectors) if the mode shapes are required. All quantities are easy to determine given a knowledge of $n$ and the simple referenced formulae [2,20-22]. This is equivalent to forming the substitute systems.

- Determine the first $j$ uncoupled frequencies of a component member with the given boundary conditions from Table 1.

-Substitute the results developed above, together with the value of $\gamma$, into Eq.(22), while noting Eq.(25). Calculate the $j$ natural frequencies corresponding to each substitute system.

- For each substitute system frequency: 
-Develop the substitute system mode shape from Eq.(16).

- Retrieve the mode shapes of the original members by multiplying the substitute system mode shapes by the appropriate components of the corresponding modal participation vector.

- Re-assemble the natural frequencies in ascending order and ensure that those of interest are bounded above in their respective substitute system.

\section{Numerical examples}

Three examples are now given that confirm the correctness and accuracy of the approach, while also giving an indication of its range of application. Example 1 and the first problem of Example 2 can be solved easily by hand using the procedure outlined above. Note that the $\lambda_{i}^{*}$ values given by the referenced formulae are equal to $r \lambda_{i} / k$, where $r$ and $k$ are the constant system values given in the table of Appendix B and the elastic interface stiffness / unit length, respectively. The remaining problems can be used to validate Eq.(22) when used in more complex problems.

\subsection{Example 1}

Consideration is first given to the simple problem of two identical and parallel taut strings of length $1 \mathrm{~m}$ that are linked by a single elastic interface of stiffness $k=200 \mathrm{~N} / \mathrm{m}^{2}$. The mass/unit length and string tension for both members are $0.01 \mathrm{~kg} / \mathrm{m}$ and $50 \mathrm{~N}$, respectively. The results are presented in Table 2, where they are compared with those of Oniszczuk [5]. It is interesting to note in this example, that since there is a line of structural symmetry parallel to the taut strings, the mode shapes can be characterised as either symmetric or antisymmetric about this axis. It can then be seen that the frequencies corresponding to antisymmetric modes are identical to the individual taut string frequencies, since the distance between corresponding points on each string must remain constant during the motion, which is therefore not influenced by the massless interface. Hence $\lambda_{0}=\lambda_{1}=0.0$. However, this is clearly not true in the case of symmetric modes. See Appendix B for equivalent taut string parameters.

Table 2: Comparison between the natural frequencies given by Oniszczuk [5] and the presented theory for the parallel string problem of Example 1. The frequencies correspond to either A/S (Anti-Symmetric) or S (Symmetric) modes about the horizontal axis of symmetry.

\begin{tabular}{ccccc}
\hline \multirow{2}{*}{$\begin{array}{c}\text { Modal } \\
\text { No. }\end{array}$} & \multicolumn{4}{c}{ Natural frequencies (rad/sec) } \\
\cline { 2 - 5 } & Oniszczuk [5] & S & $\begin{array}{c}\text { Substitute } \\
\text { system } 1\end{array}$ & $\begin{array}{c}\text { Substitute } \\
\text { system } 2 \\
\lambda_{1}=0.0\end{array}$ \\
& & & & $\lambda_{2}=8.0$ \\
& & & 222.144 & 298.911 \\
1 & $221.1^{*}$ & 298.9 & 444.288 & 487.229 \\
2 & 444.3 & 487.2 & 666.432 & 695.796 \\
3 & 666.4 & 695.8 & 888.577 & 910.806 \\
4 & 888.6 & 910.8 & 1110.72 & 1128.58 \\
5 & 1110.7 & 1128.6 & 1332.86 & 1347.80 \\
6 & 1332.9 & 1347.8 & &
\end{tabular}

* This value has been confirmed as a typing error in the original paper and should be 222.1. 


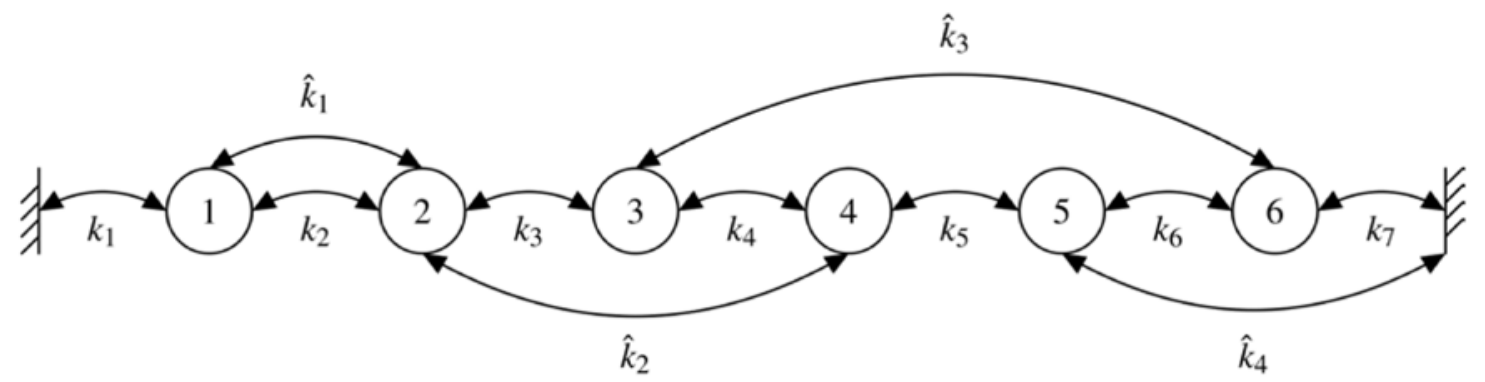

Fig. 2. Cross-section of the structure considered in Example 2. The six shafts are only able to twist about their respective longitudinal axes and are connected by uniformly distributed torsional interfaces, $k_{i}(i=1, \ldots 7)$, to neighbours and/or foundations. Similar additional interfaces, $\hat{k}_{i}(i=1, \ldots 4)$, provide cross connection between some of the shafts and a foundation.

\subsection{Example 2}

Fig. 2 shows the cross-section of a structure comprising six shafts that are linked to each other and to longitudinal foundations by uniformly distributed, elastic torsional interfaces $k_{i}$, $i=1, \ldots 7$. Additional similar interfaces, $\hat{k}_{i}, i=1, \ldots 4$, provide cross connections between some of the shafts and a foundation. (An aid to visualising such torsional interfaces is to consider them as distributed extensional interfaces that are linked to the tips of rigid, massless fins on each side of the member.) Two problems are now considered using the data given below. (See Appendix B for equivalent torsion parameters.) In the first of these, all interfaces $\hat{k}_{i}$ are assumed to have zero stiffness, while in the second they take on their presented values.

Member data: $L=4.0 \mathrm{~m}, m=175 \mathrm{~kg} / \mathrm{m}, r_{t}^{2}=0.027 \mathrm{~m}^{2}, G J=2.2 \times 10^{5} \mathrm{Nm}^{2}$ and the torsional interface stiffnesses are given in Table 3. All shafts are the same and have clamped / clamped boundary conditions. The results are given in Table 4 and clearly demonstrate that individual substitute systems do not yield sequential natural frequencies of the system.

Table 3: Torsional interface data for Example 2.

\begin{tabular}{ccc}
\hline$i$ & $k_{i} \times 10^{4}$ & $\hat{k}_{i} \times 10^{6}$ \\
& $\mathrm{~N}$ & $\mathrm{~N}$ \\
\hline & & \\
1 & 1.1 & 3.7 \\
2 & 1.3 & 3.1 \\
3 & 1.7 & 2.3 \\
4 & 1.9 & 1.9 \\
5 & 23.0 & \\
6 & 25.0 & \\
7 & 29.0 & \\
\hline
\end{tabular}




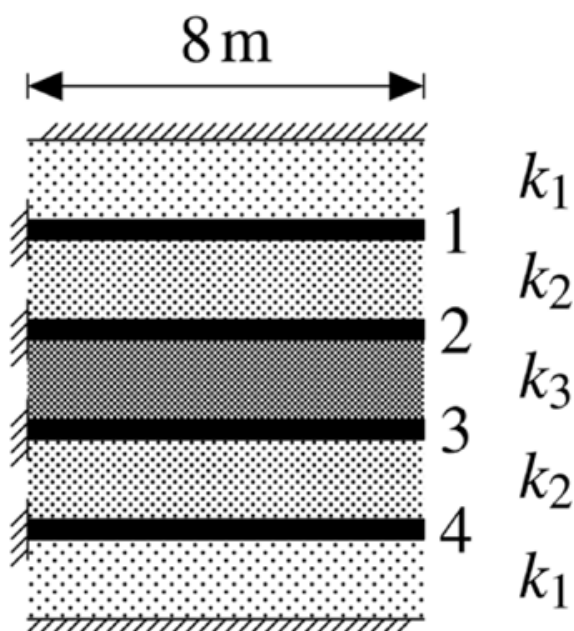

(a)
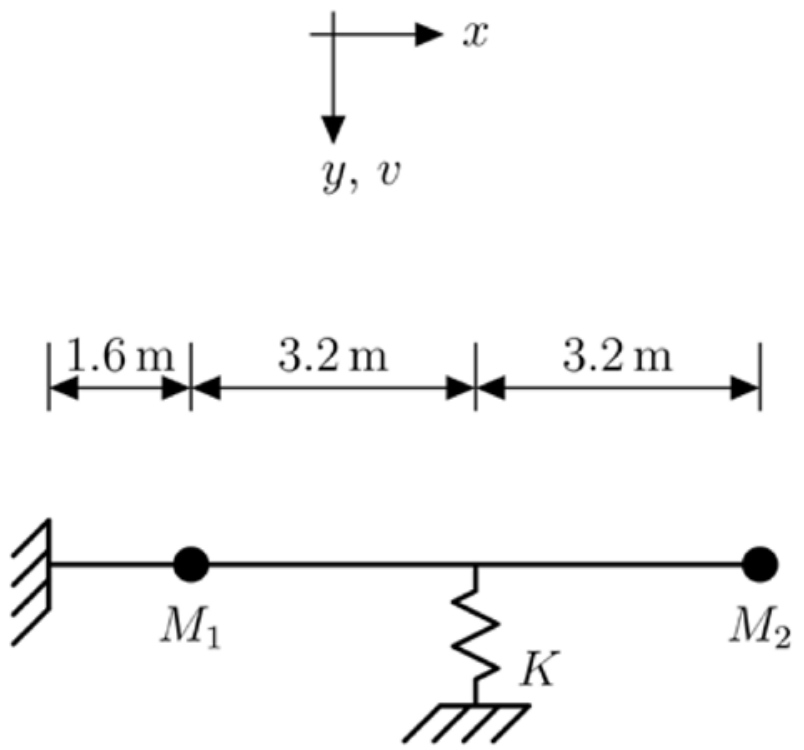

(b)

Fig. 3. The structure considered in Example 3: (a) General arrangement of idealised member and elastic interfaces; (b) The detailed form of each member idealised in (a).

Table 4: Results for the problems of Example 2.

(a) Linear eigenvalues.

\begin{tabular}{|c|c|c|c|c|c|c|}
\hline \multirow{3}{*}{$\begin{array}{c}\text { Problem } \\
\text { No. }\end{array}$} & \multicolumn{6}{|c|}{ Linear Eigenvalues } \\
\hline & \multirow[b]{2}{*}{1} & \multicolumn{3}{|c|}{ Substitute System No. } & \multirow[b]{2}{*}{5} & \multirow[b]{2}{*}{6} \\
\hline & & 2 & 3 & 4 & & \\
\hline 1 & 0.562731 & 1.91961 & 3.50989 & 5.14576 & 28.9375 & 58.7608 \\
\hline 2 & 0.597961 & 2.69006 & 5.28241 & 43.5957 & 494.669 & 541.092 \\
\hline
\end{tabular}

(b) Natural frequencies. The system frequency numbers are shown in brackets.

\begin{tabular}{|c|c|c|c|c|c|c|c|}
\hline \multirow{3}{*}{$\begin{array}{c}\text { Modal } \\
\text { No. }\end{array}$} & \multicolumn{7}{|c|}{ Natural Frequencies (Hz) } \\
\hline & \multicolumn{7}{|c|}{ Substitute System No. } \\
\hline & 1 & 2 & 3 & & 4 & 5 & 6 \\
\hline & \multicolumn{7}{|c|}{ Problem 1} \\
\hline 1 & 27.7307(1) & $29.4790(2)$ & 31.4044 & $4(3)$ & 33.2689 (4) & $53.4843(5)$ & $71.1261(11)$ \\
\hline 2 & $54.3280(6)$ & $55.2409(7)$ & 56.2919 & $9(8)$ & $57.3529(9)$ & $71.0149(10)$ & $85.0968(16)$ \\
\hline 3 & $81.1733(12)$ & $81.7870(13)$ & 82.5006 & (14) & $83.2282(15)$ & $93.1702(17)$ & $104.303(18)$ \\
\hline 4 & $108.082(19)$ & $108.544(20)$ & 109.082 & (21) & $109.634(22)$ & $117.360(23)$ & $126.379(24)$ \\
\hline 5 & 135.016 & $135.386(26)$ & 135.818 & $(27)$ & $136.261(28)$ & $142.551(29)$ & $150.064(30)$ \\
\hline & \multicolumn{7}{|c|}{ Problem 2} \\
\hline 1 & $27.7775(1)$ & $30.4270(2)$ & 33.4199 & $9(3)$ & 62.7778 & 192.849 (28) & $201.526(31)$ \\
\hline 2 & 54.3519 (4) & 55.7525 (5) & 57.4407 & $7(6)$ & 78.2534 (8) & 198.427 (30) & 206.870 \\
\hline 3 & $81.1893(9)$ & 82.1335 (10) & 83.2886 & (11) & $98.7986(12)$ & 207.391 & 215.483 \\
\hline 4 & 108.094 (13) & 108.805 & 109.679 & (15) & $121.876(16)$ & 219.325 & $226.992(40)$ \\
\hline 5 & $135.026(17)$ & 135.595 (18) & 136.298 & (19) & $146.292(20)$ & & \\
\hline 6 & $161.971(21)$ & $162.446(22)$ & 163.033 & (23) & $171.476(24)$ & & \\
\hline 7 & $188.924(25)$ & $189.332(26)$ & 189.836 & $(27)$ & 197.134 (29) & & \\
\hline 8 & $215.882(35)$ & 216.239 (36) & 216.680 & (37) & 223.102 (39) & & \\
\hline
\end{tabular}




\subsection{Example 3}

Fig. 3 shows a four member, simple shear beam system that is symmetric about a central horizontal axis. All members have the same material and geometric properties, carry the same masses and are supported in an identical fashion. The relevant data are given below.

$k_{1}=10^{2} \mathrm{~N} / \mathrm{m}^{2}, \quad k_{2}=10^{3} \mathrm{~N} / \mathrm{m}^{2}, \quad k_{3}=10^{4} \mathrm{~N} / \mathrm{m}^{2}, \quad m=90 \mathrm{~kg} / \mathrm{m}, \quad A_{s} G=6.0 \times 10^{4} \mathrm{~N}$

$M_{1}=300 \mathrm{~kg}, \quad M_{2}=200 \mathrm{~kg}, K=1.5 \times 10^{6} \mathrm{~N} / \mathrm{m}$

It is apparent from Fig. 3 that only two independent sub-sets are required to describe the structure, namely one of length $1.6 \mathrm{~m}$ and the other of length $3.2 \mathrm{~m}$. Thus the second sub-set and the data developed from it subsequently must be used twice when establishing the extended substitute systems. The results are given in Table 5.

Table 5: Results for the problem of Example 3.

(a) Linear eigenvalue problem.

\begin{tabular}{cccccc}
\hline Modal & $\lambda_{i}$ & \multicolumn{4}{c}{ Modal vector (members top to bottom) } \\
\cline { 3 - 6 } No. $i$ & & \multicolumn{5}{c}{ Set 1} \\
& & -0.951249 & -1.0 & -1.0 & -0.951249 \\
2 & 0.00208003 & 1.0 & 0.0501250 & -0.0501250 & -1.0 \\
3 & 0.0447947 & -1.0 & 0.951249 & 0.951249 & -1.0 \\
4 & 0.0875200 & -1.0 & 1.0 & -0.0501250 \\
& 0.898139 & 0.0501250 & & & \\
1 & & & Set 2 & -1.0 & -0.951249 \\
2 & 0.00832013 & -0.951249 & -1.0 & 1.0 \\
3 & 0.0179179 & -1.0 & -0.0501250 & 0.0501250 & 1.0 \\
4 & 3.59255 & -0.0501250 & -0.951249 & -0.951249 & 0.0501250 \\
\hline
\end{tabular}

(b) Natural frequencies computed using the presented theory and the method of Howson et al. [24]. A/S and S denote anti-symmetry and symmetry of the mode shape about the horizontal axis of symmetry in the structure. The system frequency numbers are shown in brackets.

\begin{tabular}{|c|c|c|c|c|c|}
\hline \multicolumn{6}{|c|}{ Exact natural frequencies (Hz) } \\
\hline & \multirow{2}{*}{\multicolumn{2}{|c|}{$\begin{array}{c}\text { Presented theory } \\
\text { Extended substitute system no. }\end{array}$}} & \multirow[b]{3}{*}{4} & \multicolumn{2}{|c|}{ Howson et al. [24] } \\
\hline & & & & \multirow[t]{2}{*}{$\mathrm{A} / \mathrm{S}$} & \multirow[t]{2}{*}{$\mathrm{S}$} \\
\hline 1 & 2 & 3 & & & \\
\hline $1.24404(1)$ & $1.283723(2)$ & $1.32181(3)$ & $1.85561(7)$ & 1.24404 (1) & $1.28373(2)$ \\
\hline 1.75435 (4) & $1.78426(5)$ & $1.81342(6)$ & 2.26128 (8) & 1.32181 & $1.78426(5)$ \\
\hline $4.31398(9)$ & $4.34214(10)$ & $4.37022(11)$ & $4.88507(15)$ & 1.75435 (4) & $1.85561(7)$ \\
\hline \multirow[t]{5}{*}{$4.49715(12)$} & $4.52292(13)$ & $4.54866(14)$ & $5.02719(16)$ & $1.81342(6)$ & $2.26128(8)$ \\
\hline & & & & 4.31398 (9) & $4.34214(10)$ \\
\hline & & & & $4.37022(11)$ & 4.52292 (13) \\
\hline & & & & $4.49715(12)$ & 4.88507 (15) \\
\hline & & & & $4.54866(14)$ & $5.02719(16)$ \\
\hline
\end{tabular}


(c) Mode shapes of the original members for two representative natural frequencies computed using the presented theory and the method of Howson et al. [24]. The system frequency numbers are shown in brackets.

\begin{tabular}{|c|c|c|c|c|c|}
\hline \multirow{2}{*}{$\begin{array}{c}\text { Frequency } \\
(\mathrm{Hz})\end{array}$} & \multirow{2}{*}{$\begin{array}{l}\text { Member } \\
\text { no. } i\end{array}$} & \multicolumn{4}{|c|}{ Structure node numbers } \\
\hline & & 1 & 2 & 3 & 4 \\
\hline \multirow{12}{*}{$\begin{array}{c}1.24404 \\
(1)\end{array}$} & & \multicolumn{4}{|c|}{ Extended system mode shape } \\
\hline & 1 & 0.0 & -0.0111651 & -0.0145907 & -1.0 \\
\hline & \multicolumn{5}{|c|}{ Mode shape of original member $i$ using the presented method } \\
\hline & 1 & 0.0 & 0.0106207 & 0.0138794 & 0.951249 \\
\hline & 2 & 0.0 & 0.0111651 & 0.0145907 & 1.0 \\
\hline & 3 & 0.0 & 0.0111651 & 0.0145907 & 1.0 \\
\hline & 4 & 0.0 & 0.0106207 & 0.0138794 & 0.951249 \\
\hline & & \multicolumn{4}{|c|}{$\begin{array}{l}\text { Mode shape of original member } i \text { by the method of Howson et al. } \\
\text { (2012) }\end{array}$} \\
\hline & 1 & 0.0 & -0.0106208 & -0.0138794 & -0.951249 \\
\hline & 2 & 0.0 & -0.0111651 & -0.0145907 & -1.0 \\
\hline & 3 & 0.0 & -0.0111651 & -0.0145907 & -1.0 \\
\hline & 4 & 0.0 & -0.0106208 & -0.0138794 & -0.951249 \\
\hline 2.26128 & & \multicolumn{4}{|c|}{ Extended system mode shape } \\
\hline (8) & 2 & 0.0 & 1.0 & 0.0110912 & 0.0102888 \\
\hline \multicolumn{6}{|c|}{ Mode shape of original member $i$ using the presented method } \\
\hline & 1 & 0.0 & 0.0501250 & $5.46984 \mathrm{E}-4$ & $-5.15724 \mathrm{E}-4$ \\
\hline & 2 & 0.0 & -1.0 & -0.0110912 & 0.0102888 \\
\hline & 3 & 0.0 & 1.0 & 0.0110912 & -0.0102888 \\
\hline & 4 & 0.0 & -0.0501250 & $-5.46984 E-4$ & $5.15724 \mathrm{E}-4$ \\
\hline & & \multicolumn{4}{|c|}{$\begin{array}{l}\text { Mode shape of original member } i \text { by the method of Howson et al. } \\
\text { (2012) }\end{array}$} \\
\hline & 1 & 0.0 & 0.0501250 & 5.55938E-4 & $-5.15847 E-4$ \\
\hline & 2 & 0.0 & -1.0 & -0.0110912 & 0.0102886 \\
\hline & 3 & 0.0 & 1.0 & 0.0110912 & -0.0102886 \\
\hline & 4 & 0.0 & -0.0501250 & $-5.55938 \mathrm{E}-4$ & $5.15847 \mathrm{E}-4$ \\
\hline
\end{tabular}

Section (a) of Table 5 gives the eigenvalues and corresponding modal vectors stemming from the linear eigenvalue problem posed by the two independent sets of members. Section (b) gives the natural frequencies by extended substitute system and the corresponding values computed by the method of Howson et al. [24]. Section (c) considers two representative frequencies and presents their corresponding mode shapes calculated from the appropriate extended substitute system i.e. the first for the lower frequency and the fourth for the higher frequency. These mode shapes then yield the mode shapes of the original members, according to Section 2.6.1, as follows. Consider the extended system mode shape corresponding to $1.24404 \mathrm{~Hz}$. Then the mode shape of original member $i$ is given by 
multiplying the extended system mode shape by the $i^{\text {th }}$ element of the modal vector corresponding to $\lambda_{i}$, which is given in Section (a) of the results.

\section{Discussion and Conclusions}

Consideration has been given to determining the exact natural frequencies and modes of vibration of a class of structures comprising any number of related parallel members that are linked to each other, and possibly also to foundations, by uniformly distributed elastic interfaces of unequal stiffness. The members themselves are considered to have a uniform distribution of mass and stiffness and their motion is governed by a second order SturmLiouville equation. This encompasses a family of simple, but extremely useful structural elements and enables the motion of strings and shear beams, together with the axial and torsional motion of bars to be described exactly.

The approach adopted considers two forms of solution. The first of these is general and reduces the solution of an $n$ member system to the solution of $n$ substitute systems that together describe the complete motion of the original structure exactly. Each substitute system is described by an exact dynamic stiffness matrix of order two and can be solved simply for any possible boundary conditions using the Wittrick-Williams algorithm, thus guaranteeing convergence upon any required natural frequency to any desired accuracy with the certain knowledge that none have been missed. Furthermore, adoption of the stiffness approach enables the original structure to be dissected into exact finite elements that subsequently enable additional point masses and in-span point supports to be accounted for.

The second form of solution utilises the same theory and with little loss of generality enables a useful range of practical problems to be solved by hand. The process is described in detail for a typical repetitive structure in which all the members are identical with classical support conditions, there are no in-span point masses or point supports and all elastic interfaces are the same with no cross-connections. In such cases, exact hand solution can be achieved easily using simple referenced formulae in combination with an equally simple, but comprehensive relationship between groups of natural frequencies.

Finally, the theory has been developed in the context of linked shear beams. This leads directly to a practical application of the method in which an approximate analysis can be undertaken to assess the vibrational characteristics of sway frames either linked together or additionally linked to shear cores. This can be achieved using well established simplification procedures that reduce multi-bay, multi-storey sway frames to equivalent one bay frames and then to simpler global models that can often retain sufficient accuracy for preliminary analysis and design procedures.

\section{References}

[1] S.S. Rao, Vibration of Continuous Systems, John Wiley and Sons, New Jersey, 2007.

[2] F.W. Williams, Simple buckling and vibration analyses of beam or spring connected structures, Journal of Sound and Vibration 62 (1979) 481-491.

[3] Q. Mao, Free vibration analysis of elastically connected multiple-beams by using the Adomiam modified decomposition method, Journal of Sound and Vibration 331 (2012) 2532-2542.

[4] Z. Oniszczuk, Transverse Vibrations of Elastically Connected DoubleString Complex System, Part I: Free Vibrations, Journal of Sound and Vibration 232 (2000) 355-366. 
[5] Z. Oniszczuk, Transverse Vibrations of Elastically Connected DoubleString Complex System, Part II: Forced Vibrations, Journal of Sound and Vibration 232 (2000) 367-386.

[6] Z. Oniszczuk, Damped Vibration Analysis of an Elastically Connected Complex Double String System, Journal of Sound and Vibration 264 (2003) 253-271.

[7] J. Rusin, P. Sniady, P. Sniady, Vibrations of Double-String Complex System Under Moving Forces. Closed Solutions, Journal of Sound and Vibration 330 (2011) 404-415.

[8] H. Erol, M. Gurgoze, Longitudinal Vibrations of a Double-Rod System Coupled by Springs and Dampers, Journal of Sound and Vibration 276 (2004) 419-430.

[9] S. Kukla, J. Przybylski, L. Tomski, Longitudinal Vibration of Rods Coupled by Translational Springs, Journal of Sound and Vibration 185 (1995) 717-722.

[10] Q.S. Li, G.Q. Li, D.K. Liu, Exact Solutions for Longitudinal Vibration of Rods Coupled by Translational Springs, International Journal of Mechanical Sciences 42 (2000) 1135-1152.

[11] H-P. Lin, S-C Chang, Free Vibrations of Two Rods Connected by MultiSpring-Mass Systems, Journal of Sound and Vibration 330 (2011) 25092519.

[12] C.N. Bapat, N. Bhutani, General Approach for Free and Forced Vibrations of Stepped Systems Governed by the One-Dimensional Wave Equation with Non-Classical Boundary Conditions, Journal of Sound and Vibration 172 (1994) 1-22.

[13] S.A. Nayfeh, K.K. Varanasi, A model for the damping of torsional vibration in thin-walled tubes with constrained viscoelastic layers, Journal of Sound and Vibration 278 (2004) 825-846.

[14] G.R. Bhashyam, G. Prathap, The second frequency spectrum of Timoshenko beams, Journal of Sound and Vibration 76 (1981) 407-420.

[15] W.P. Howson, F.W. Williams, A Unified Principle of Multiples for Lateral Deflection, Buckling and Vibration of Multi-Storey, Multi-Bay Sway Frames, Proceedings of the Second International Conference on Advances in Steel Structures, Elsevier, Oxford, Vol 1 (1999) 87-98.

[16] B. Rafezy, W.P. Howson, Natural Frequencies of Plane Sway Frames: An Overview of Two Simple Models, in Proceedings of the International Conference on Computational and Experimental Engineering and Sciences, Tech Science Press, Duluth, Georgia, USA, Paper 339, 2003.

[17] W.P. Howson, Global Analysis: Back to the Future, The Structural Engineer 84 (2006) 18-21.

[18] M. Sato, S. Kanie, T. Mikami, Mathematical analogy of a beam on elastic supports as a beam on an elastic foundation, Applied Mathematical Modelling 32(5) (2008) 688-699.

[19] W.P. Howson, A compact method for computing the eigenvalues and eigenvectors of plane frames, Advances in Engineering Software, 1 (1979) 181-190.

[20] R.T. Gregory, D.L. Karney, A collection of matrices for testing computational algorithms, Wiley Interscience, London 1969.

[21] W-C Yueh, Eigenvalues of several tridiagonal matrices, Applied Mathematics E-Notes 5 (2005) 66-74. 
[22] S. Kouachi, Eigenvalues and eigenvectors of tridiagonal matrices, Electronic Journal of Linear Algebra 15 (2006) 115-133.

[23] W.P. Howson, F.W. Williams, Natural frequencies of frames with axially loaded Timoshenko members, Journal of Sound and Vibration 26 (1973) 503-515.

[24] W.P. Howson, A. Watson, B. Rafezy, Exact dynamic stiffness matrix for a class of elastically supported structures. Proceedings of the Twelfth International Conference on Computational Structures Technology, Dubrovnik, Croatia. Civil-Comp Press, (2012) pp. 16.

\section{Appendix A}

\section{A.1. Formulae for the Wittrick-Williams algorithm}

The Wittrick-Williams root counting algorithm has been available for well over thirty years and the following formula can be established easily from many sources, such as reference [23]. In the current notation and context, it states that the number of coupled natural frequencies passed by a trial frequency, $\omega^{*}$, is given by $J\left(\omega^{*}\right)$ where

$$
J\left(\omega^{*}\right)=0 \quad \text { for } \quad \chi_{i}^{2}<0
$$

and

$$
J\left(\omega^{*}\right)=\operatorname{int}\left(\chi_{i} / \pi\right)+s\left\{\mathbf{K}_{s i}\right\} \quad \text { for } \quad \chi_{i}^{2}>0
$$

where int( ) is the highest integer less than the bracketed term and $s\left\{\mathbf{K}_{s}\right\}$ is the sign count of the substitute system matrix, which is equal to the number of negative elements on the leading diagonal of the upper triangular matrix obtained from $\mathbf{K}_{s i}$, when $\omega=\omega^{*}$, by the standard form of Gauss elimination without row interchanges.

\section{Appendix B}

\section{B.1. Corresponding member properties}

The theory presented in the body of this paper is written in the context of a simple shear beam. However, it applies additionally to the elastically supported motion of a range of useful elements including pure shear beams [14], strings and the axial and torsional motion of bars, see reference [1]. In order to implement these alternative relationships, it is assumed that the members are always arranged in an analogous way to those described herein, but consistent with the member type, and that the distributed elastic interfaces resist the motion in the appropriate freedom. Eq.(4), the constitutive equation, should then be replaced by the appropriate one from Table B1. These equations, plus the symbols $r_{i}$ and $\gamma_{i}$ that should be used in Eq.(3) and subsequently, are described in terms of symbols that are commonly used to represent the necessary parameters and whose meanings are straightforwardly deduced by analogy with the simple shear case. 
Table B1: Key relationships for alternative member types. $m$ is the mass / unit length, $r_{b}$ and $r_{t}$ are the bending and twisting (polar) radius of gyration, respectively, $T$ is the string tension and $A_{s} G, E A, E I$ and $G J$ are the effective shear, axial, bending and torsional rigidities, respectively.

\begin{tabular}{cccc}
\hline Member type & $\begin{array}{c}\text { Constitutive } \\
\text { Equation }\end{array}$ & $r_{i}$ & $\gamma_{i}$ \\
\hline Simple shear beam & $Q_{i}=r_{i} L d V_{i} / d \xi$ & $A_{s} G_{i} / L^{2}$ & $m_{i} / r_{i}$ \\
Pure shear beam & $M_{i}=-r_{i} L d \Psi_{i} / d \xi$ & $E I_{i} / L^{2}$ & $\left(r_{b}^{2} m_{i}-A_{s} G_{i} / \omega^{2}\right) / r_{i}$ \\
String & $Q_{i}=r_{i} L d V_{i} / d \xi$ & $T_{i} / L^{2}$ & $m_{i} / r_{i}$ \\
Bar (Axial) & $F_{i}=r_{i} L d U_{i} / d \xi$ & $E A_{i} / L^{2}$ & $m_{i} / r_{i}$ \\
Bar (Torsional) & $\tilde{T}_{i}=r_{i} L d \Phi_{i} / d \xi$ & $G J_{i} / L^{2}$ & $r_{t}^{2} m_{i} / r_{i}$ \\
\hline
\end{tabular}

